\title{
Unusual presentation of a large pituitary tumour in relation to diving
}

\author{
A. Magid O. Bakheit and P.G.E. Kennedy \\ Glasgow University Department of Neurology, Institute of Neurological Sciences, Southern General Hospital, \\ Glasgow G51 4TF, UK.
}

\begin{abstract}
Summary: A case of necrosis of a pituitary tumour occurring in the context of diving is described. The presenting features and subsequent course suggested a brain stem vascular event. The tumour was not detected by routine computerized tomographic scanning, but was identified with magnetic resonance imaging. The possible pathophysiological mechanism is discussed.
\end{abstract}

\section{Introduction}

Pituitary tumours present typically with slowly progressive neurological and endocrine deficits, and are usually detectable on computed tomographic (CT) scanning. We describe here a patient with a large pituitary tumour who presented with acute symptoms and signs suggestive of brain-stem dysfunction in the absence of tumour extension or hydrocephalus. Although routine CT scanning was normal the tumour was subsequently detected by magnetic resonance imaging (MRI) scanning. Interestingly, the patient's symptoms also had a temporal relationship to diving.

\section{Case report}

A 53 year old man took up sea diving as a hobby in November 1987. A medical examination prior to this was normal. He was admitted on 5 April 1988, with severe headache, nausea, diplopia and hiccups which had started 10 days prior to admission, and 90 minutes following a 10 metre dive which had lasted for 20 minutes. The headache, which he described as a tight band around his head, was exacerbated by bending forwards, coughing and sneezing. He also vomited twice and had felt generally unwell. Four days later he developed horizontal diplopia with the image separation maximal to the right. His symptoms remained static until his admission to hospital, but started to improve gradually over the following week. He lost $18 \mathrm{lb}$ in weight during the first two weeks of his illness.

Six weeks previously he had experienced a similar

Correspondence: Professor P.G.E. Kennedy, M.D., Ph.D., F.R.C.P.

Accepted: 26 July 1988 headache, and vomited twice following a 12 metre dive lasting for $\mathbf{4 0}$ minutes. His symptoms on this occasion started about one hour after the dive. These symptoms resolved spontaneously over 4 days.

In the intervening period between these two episodes the patient dived 5 times to a depth of 18 metres for 30 minutes on each occasion without ill-effects.

On examination there was no neck stiffness. He was apathetic but fully orientated. He had a $2 \mathrm{~mm}$ ptosis of the left eye. Both pupils were equal and regular, but the left pupillary reaction to light was sluggish. Adduction of the left eye was limited. The signs were thought to indicate a partial left third nerve palsy. In addition, vertical gaze was impaired and there was coarse jerk nystagmus on lateral gaze. His visual acuity, visual fields and fundi were normal. Muscle tone, power and coordination were also normal in all limbs. His tendon reflexes were symmetrical. The right plantar response was absent and the left extensor. All sensory modalities were intact.

Full blood count, ESR biochemical screening, and serological tests for syphilis were normal or negative as were liver ultrasound, chest X-ray and endocrinology screening. Doppler carotid ultrasound did not demonstrate any haemodynamically significant extracranial carotid artery disease. Routine CT tomoscan was normal. An MRI scan showed an intrasellar tumour $2 \mathrm{~cm}$ in diameter extending into the sphenoid sinus (Figure 1). A plain lateral skull X-ray confirmed enlargement of the pituitary fossa, loss of definition of the floor of the sella turcica and erosion of the dorsum sellae (Figure 2). A coronal CT scan showed a large intrasellar tumour with downward extension into the sphenoid sinus (Figure 3). Visual field perimetry was normal.

The Fellowship of Postgraduate Medicine, 1989 


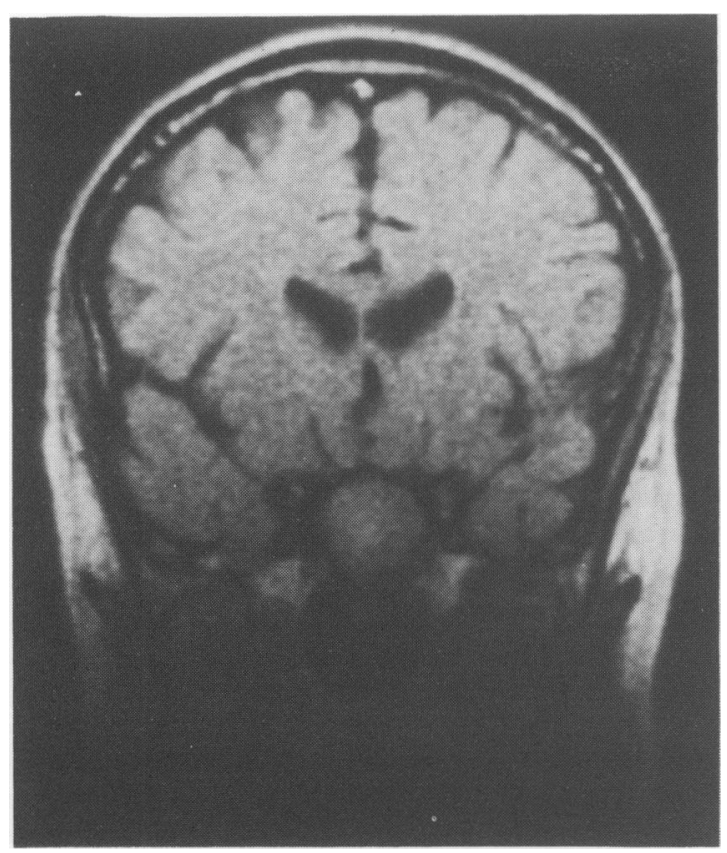

Figure 1 MRI showing a large intrasellar tumour extending into the sphenoid sinus.

Surgical removal of a pituitary tumour was carried out through a trans-sphenoidal approach (Professor G.M. Teasdale). The tumour consisted almost entirely of structureless tissue due to a vascular necrosis. On microscopy there was one small group of small tumour cells whose appearances were those of a carcinoma.

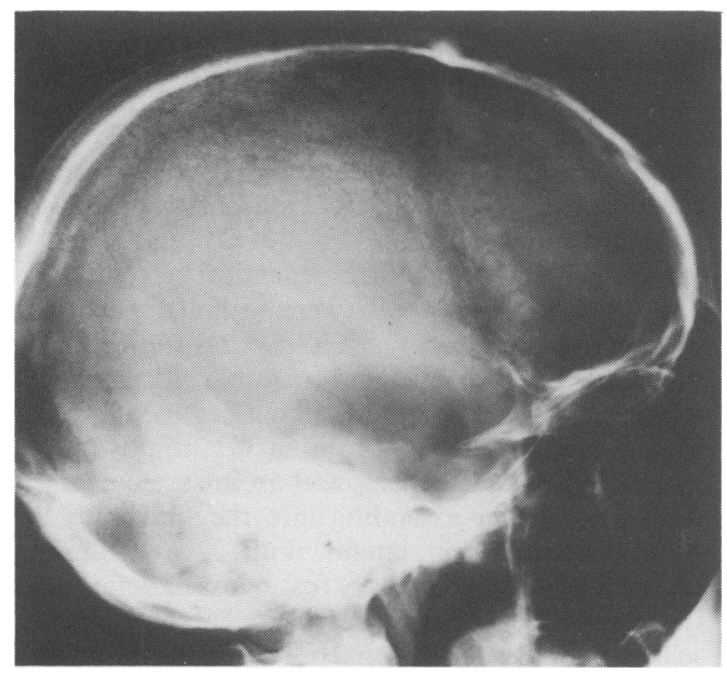

Figure 2 Skull X-ray (lateral view). Note enlargement of pituitary fossa, loss of definition of the floor of the sella and erosion of the posterior clinoid.

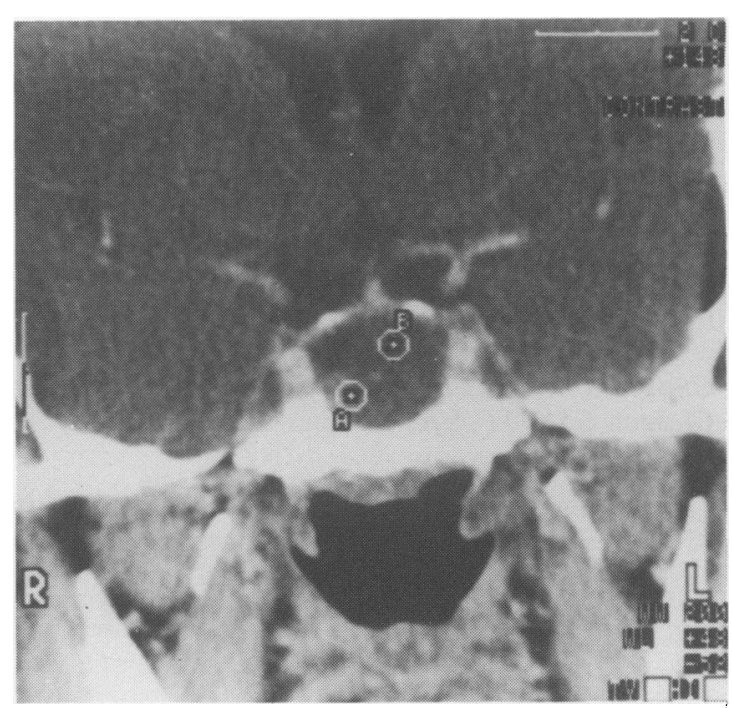

Figure 3 Coronal CT scan demonstrating the extension of an intrasellar tumour into the sphenoid sinus. Tumour mass is marked A and B.

Discussion

The classical presentation of an intrasellar tumour is with a slowly evolving syndrome of chiasmal compres sion and various endocrine abnormalities. However; isolated or multiple cranial nerve palsies may be the earliest manifestation of these tumours antedating other symptoms and signs by many weeks or even months. ${ }^{1}$ Total ophthalmoplegia usually occurs suddenly, whereas isolated cranial nerve palsies tend to develop gradually. ${ }^{2}$ The commonest isolated cranial nerve palsy is that of the third nerve which is compressed between the tumour and the interclinoid ligaments at the point where it enters the cavernous sinus. ${ }^{3}$ Acute ocular palsies result from stretching of nerve structures which follows a sudden increase in the tumour size due to haemorrhage or massive necrosis. The presence of a unilateral ocular palsy in the absence of a visual field defect is a rare, but well recognized presenting feature of pituitary tumours ${ }^{4}$ and was an early sign in our patient.

The neurological manifestations of intrasellar tumours largely depend on the direction of tumour expansion and, to a lesser extent, the tumour's histological characteristics. Upward tumour expansion causes bitemporal visual field loss, while anterior extension compresses the prechiasmal optic nerve leading to decreased visual acuity, sluggish pupillary reaction to light and eventually optic atrophy. Exten- 
sion of an intrasellar mass downwards into the sphenoid sinuses causes nasal discharge. Less commonly the tumour extends backwards causing brain stem, pyramidal tract and cerebellar signs. Surprisingly, our patient developed a partial third nerve palsy and brain stem features such as nystagmus, impaired vertical gaze, persistent nausea and hiccups in the absence of posterior extension of the tumour or hydrocephalus.

Headache and vomiting are common features of acute massive tumour necrosis and could occur through a sudden rise in intracranial pressure due to tumour expansion or, alternatively, by meningeal irritation caused by the leaked tumour necrotic tissue. Neither of these explanations seems likely in our case since there was no papilloedema or neck stiffness. However, the raised pressure type of headache experienced by our patient is presumably due to extension of the tumour beyond the diaphragm sellae. The apathy and slow cerebration which we observed may have been secondary to compression of the floor of the third ventricle and hypothalamus. ${ }^{5}$

Although small areas of tumour necrosis are a common autopsy finding, massive pituitary infarction is rare but well-recognized. It may occur spontaneously, ${ }^{6,7}$ after head injury ${ }^{8}$ or following radiotherapy. ${ }^{9}$ To the best of our knowledge massive necrosis of pituitary tumour has not been reported before in association with barotrauma.

Barometric pressure changes at any depth (or altitude) occur in a predictable fashion and are readily available from barometric pressure charts. At a depth of 10 metres, for example, the barometric pressure is two atmospheres, at 20 metres - three atmospheres and at 30 metres - four atmospheres. During diving these atmospheric pressure changes are transmitted to the gas-containing body cavities (middle ear, paranasal sinuses and respiratory tract). An equilibrium between these cavities and the environment is reached if there is free gas movement between the two, and under these circumstances no pressure differential is created and ill-effects from diving are unlikely to be experienced. If gas movements are reduced during descent, however, a relative vacuum is created with resulting hyperaemia and congestion of the mucosal membranes and rupture of the microvasculature. Further, if gas is trapped in body cavities (e.g. due to obstruction by a tumour) during ascent a high pressure gradient develops because of the rapid fall of environmental pressure relative to that of body

\section{References}

1. Weinberger, L.M., Alder, F.H. \& Grant, F.C. Pituitary adenoma and the syndrome of the cavernous sinus. Arch Ophthalmol 1940, 24: 1197. cavities. The net result is a sudden rise of intra-arterial pressure and blood vessels may rupture. We suggest that in our patient the tumour extension into the sphenoid sinus prevented the escape of gas from the latter cavity during ascent to the surface, thus creating a large pressure gradient and precipitating a massive bleed into the tumour. It is difficult, though, to explain the delayed onset of symptoms following submergence.

The greatest volume/pressure changes in gases trapped in body cavities occur with diving to shallow depths. Ascent from 30 metres, for example, results in doubling of the intracavity pressure, while ascent from 10 metres only results in similar pressure changes. ${ }^{10}$ This could explain why there were no symptoms in our patient following dives of 18 metres, but a pituitary infarction had occurred after a shallow dive.

Primary carcinoma of the pituitary (also known as Stage III adenoma, malignant adenoma or invasive adenoma) comprises $4-10 \%$ of pituitary adenomas. ${ }^{11}$ The pituitary is also a common site for metastatic carcinoma, especially from breast carcinoma. ${ }^{12}$ In the absence of evidence of extracranial malignant disease, it is most likely that our patient had a primary pituitary carcinoma.

The pituitary tumour in our case was not detected on routine CT scanning but was visualized by MRI. This highlights the value of the latter investigation in diagnosing intracranial pathology in the presence of a normal CT scan. This is consistent with the observation of Banna et al. who evaluated the diagnostic role of CT scans in pituitary tumours in a large series of cases. ${ }^{13}$ They found that only two out of $30 \mathrm{mic}-$ roadenomas (diameter less than $1 \mathrm{~cm}$ ) were detectable on CT scanning. Twenty two per cent of larger tumours were also missed. This case illustrates the importance of pursuing the neuroradiological investigations beyond CT scanning when there remains diagnostic doubt and a clinical suspicion of a structural intracranial lesion is still entertained. MRI scanning should be considered as a potentially valuable diagnostic adjunct in the diagnosis of pituitary lesions.

\section{Acknowledgements}

The help of our neuroradiological and neurosurgical colleagues is warmly appreciated. We thank Mrs M. McColl for typing the manuscript.

2. Jefferson, M. \& Rosenthal, F.D. Spontaneous necrosis in pituitary tumours (pituitary apoplexy). Lancet 1959, i: $342-344$. 
3. Cairns, H.W.B. Peripheral ocular palsies from the neurosurgical point of view. Trans Ophthalmol Soc UK 1938, 58: 464.

4. Symonds, C. Ocular palsy as the presenting symptom of pituitary adenoma. Bull Johns Hopkins Hosp 1962, 111: 72.

5. White, J.C. \& Cobb, S. Psychological changes associated with giant pituitary neoplasms. Arch Neurol Psychiat 1955, 74: 383.

6. Aitken, R.S. \& Russell, D.S. A case of Simmond's disease. Lancet 1934, ii: 802-806.

7. Tsitsopoulos, P., Andrew, J. \& Harrison, M.J.G. Pituitary apoplexy and haemorrhage into adenomas. Postgrad Med J 1986, 62: 623-626.

8. Wagenen, W.P. van. Haemorrhage into a pituitary tumour following trauma. Ann Surg 1932, 95: 625-628.
9. Dott, N.M., Bailey, P. \& Cushing, H. A consideration of the hypophyseal adenomata. Br J Surg 1925 13: 314-366.

10. Davis, J.C. Alterations in atmospheric pressure. In: Beeson, P.B., McDermott, W. \& Wyngaarden, J.B. (eds) Cecil Textbook of Medicine, 15th edition. W.B. Saunders, Philadelphia. 1979, pp. 97-103.

11. Feiring, E.H., Davidoff, L.M. \& Zimmerman, H.M. Primary carcinoma of the pituitary. $J$ Neuropathol Exp Neurol 1953, 12: 205-223.

12. Roessman, U., Kaufman, B. \& Friede, R.L. Metastic lesions in the sella turcica and pituitary gland. Cancer 1970, 25: 478-480.

13. Banna, M., Baker, H.L. \& Howser, O.W. Pituitary and parapituitary tumours on computed tomography. $\mathrm{Br} J$ Radiol 1980, 53: 1123-1143.

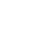

